\title{
Outcome Analysis of Anticoagulant Therapy in Critical Care Unit: The Need for a Pharmacy Managed Anticoagulant Service
}

\author{
Nwafor $\mathrm{IA}^{1 *}$, Nwafor $\mathrm{MN}^{2}$, Gbenimachor $\mathrm{MN}^{2}$, Onodugo $\mathrm{CI}^{2}$, Anakwe $\mathrm{RC}^{3}$ and Nwagha $\mathrm{TU}^{4}$ \\ ${ }^{1}$ Senior Lecturer and Honorary Consultant Cardiothoracic Surgeon, Nigeria \\ ${ }^{2}$ Deputy Director and Clinical pharmacists, ICU pharmacy, Nigeria \\ ${ }^{3}$ Senior Lecturer and Honorary Consultant Cardiologist, Nigeria \\ ${ }^{4}$ National Cardiothoraic Center of Excellence (NCTCE), Nigeria
}

*Corresponding author: Nwafor, IA, Senior Lecturer and Honorary Consultant Cardiothoracic Surgeon, Nigeria

\begin{abstract}
Background: In our center, NCTCE, there are 2 intensive care units (general and cardiac), where critically ill patients are admitted and managed. Anticoagulation is usually indicated in such patients for atrial fibrillation, valve replacement and DVT prophylaxis.

Objective: To determine the role of a clinical pharmacist in the therapeutic and clinical appraisal of such patients on anticoagulation.

Materials and Method: Over a 2-year period (June 2015 -May 2017), a study design to determine the role of a clinical pharmacist in anticoagulant treatment was made in both ICU. This was a prospective longitudinal study. Here, demography, the anticoagulation on which interventions were made on, duration of admission, areas and determinants of a clinical pharmacist's interventions were incorporated. Others included, were levels of intervention, acceptance and rejection of the interventions as well as the outcome.
\end{abstract}

Results: During the study period, 168 patients were used. Male (72) and female (96), with a female to male ratio of 1:1.3. Indications for anticoagulation were DVT prophylaxis (14.88\%), valves (83.90\%) and AF (1.10\%). Among the anticoagulants on which interventions were made on, warfarin was the highest (53.57\%) while heparin was the least (2.90\%). Duration of admission ranged from 2-40 days. Areas of intervention were on effectiveness of therapy (18.70\%), drug-drug interactions (16.43\%), adverse events (32.86\%), dosage (11.05\%), Kidney function (1.70\%), adherence (6.70\%) and drug selection (2.27\%). Levels of intervention were at prescriber $(30.51 \%)$, drug (38.56\%), patient $(25.42 \%)$ and laboratory $(5.51 \%)$. The outcome was that majority of patients (78.74\%) had their problems resolved.

Conclusion: Anticoagulation therapy of patients in ICU requires precise damaging, monitoring and appropriate patient education so as to ensure that patients benefit maximally. A clinical pharmacist has a vital role to play in the above areas.

Abbreviations: ICU: Intensive Care unit; PhRs: Pharmacist Recommendations; INR: International Normalized Ratio; ACCP: American Critical Care Physician; ADES: Adverse Events; VTE: Venous Thromboembolism; DTPS: Drug Therapies; DDI/DFI: Drug Drug/Dug Food Interactions; LMWH: Low Molecular Weight Heparins; CVP: Central Venous Pressure; CHADS: Congestive Heart failure, Hypertension, Age, Diabetes, Stoke 


\section{Introduction}

Intensive Care Unit is a unit of the hospital where critically ill patients are treated by specially trained staff (ICU-Multidisciplinary team), comprising of doctors, nurse specialists, respiratory therapists, clinical pharmacists, physiotherapists, dieticians, theatre technicians, biomedical engineers and social workers. In our center, there are 2 ICU: -

a) The general ICU for general surgery, orthopedic, neuro-, obstetrics, thoracic and trauma surgical and medical patients.

b) The cardiac ICU for perioperative open-heart surgery patients as well as those patients with myocardial infarction and acute coronary syndromes.

Critical care pharmacy services in the ICU have expanded from traditional dispensing responsibilities to being recognized as an essential component of multidisciplinary care for critically ill patients. Augmented by technology and resource utilization, this shift in roles has allowed clinical pharmacists to provide valuable services in the form of assisting physicians and clinicians with pharmacotherapy decision making, reducing medication errors and improving medication safety systems to optimize patient outcomes. Documented improvements in the management of infections, anticoagulant therapy, sedation, and analgesia for patients receiving mechanical ventilation and in emergency response help to justify the need for clinical pharmacy services for critically ill patients [1]

ICU patients have similar general risk factors for venothrombosis and pulmonary embolism as well as arterial thromboembolism manifesting as stroke, mesenteric vascular occlusion and peripheral acute limb ischemia, with other patients (age, obesity, immobilization, past personal/family history of venothrombembolism, sepsis, cancer, stroke, respiratory, heart failure, pregnancy, trauma or recent surgery) [2-4]. In addition, they have other peculiar risk factors as a result of their illnesses and/ or treatment (vasopressor use, respiratory failure, heart failure, pharmacologic sedation, mechanical ventilation, central venous pressure (CVP) catheter and end stage renal failure) [3,5]. CVP catheter in the femoral, subclavian, internal jugular and superior vena cava veins have catheter related thrombosis occurrence rate ranging from $10-69 \%, 2-10 \%, 40-56 \%$ and $7-17 \%$ respectively [2,5-7]. There is also 4-fold increased risk of pulmonary embolism from lower limb DVT in ICU patients [8]. Catheter related VTE risks increases proportionate to the duration of placement and if patient is not on LMWH anticoagulant [9]. Sepsis induce procoagulant status and favor catheter related VTE [6,10]. Vasopressor treatment is not an independent risk factor for VTE [5], however it is still linked with decreased absorption of subcutaneous heparin due to the vasoconstriction of peripheral blood vessels [11]. Platelet transfusion and high platelet levels, a common finding in ICU patients are risk factors for VTE [12]. Pharmacologic sedation is not an independent risk factor for VTE, however, when used alongside mechanical ventilation, it is. Mechanical ventilation by decreasing venous return and requiring sedation and immobilization is a risk for VTE, however, critical ill patients requiring long mechanical ventilation was compared with those who did not and the relationship between duration of mechanical ventilation and VTE was not established [2].

The implantation of an artificial heart valves exposes the patient to an increased risk of valve thrombosis and embolism; thus life- long oral anticoagulation is imperative [13]. The factors that lead to increased thromboembolism in such patients are the number of valves implanted, types of valves implanted (more in ball and cage variety), atrial fibrillation, left atrial enlargement, left ventricular dysfunction, clotting disorder and previous embolic events [14]. Atrial fibrillation is the most common arrhythmia in patients admitted in intensive care units (ICUs) and is associated with increased morbidity and mortality $[15,16]$. The problems of atrial fibrillation are low cardiac output, heart failure, hypotension and organ dysfunction as well as thromboembolism. CHADS algorithm defines the risk factors, which many critically ill patients have [17]. In addition, patients with rheumatic heart diseases, prosthetic heart valves, prio thromboembolism and persistent atrial thrombus detected by TEE as well as AF with complex atherosclerotic aortic plaque are considered at highest risk of embolic stroke if not on thromboprophylaxis [18]. In our ICU, the clinical pharmacist supervises the prescribed anticoagulant, dispenses them, monitors adverse effects, drug-drug interactions as well as compliance. These factors among others have a great role to play in the successful thromboprophylaxis and treatment of VTE and prevention of anticoagulant induced bleeding including arterial thromboembolism on account of prosthetic mechanical heart valves and atrial fibrillation.

\section{Materials and Method}

Overa2-yearperiod (June2015-May2017), a studywasdesigned to determine the role of a clinical pharmacist in anticoagulant treatment was made in both general and cardiac ICU of a tertiary hospital in Nigeria, a developing country. This was a prospective study. Here, demography, types of anticoagulation intervention was made on, duration of admission, areas and determinants of a clinical pharmacist's interventions were incorporated. Others included were levels of intervention, acceptance and rejection of the interventions as well as the outcome. The general ICU admits critical general, orthopedic, neuro-, thoracic, trauma surgical and medical patients. The cardiac ICU admits perioperative open-heart surgical patients including patients with myocardial infarction and acute coronary syndromes. The data were analyzed using Microsoft Excel 2010 and presented as frequencies and percentages using tables and charts.

The study design was approved by the hospital ethical committee. 


\section{Results}

Figure 1 shows the age ranges of patients admitted during the study period. Highest range is 31-40 years. Least is 81-90 years. Also Figure 2 shows additional indications for anticoagulation, with mechanical prosthetic heart valves having the highest number while atrial fibrillation takes the least. Table 1 shows the anticoagulants administered and the number of interventions made on them. The highest number of interventions was made on warfarin, followed by enoxaparin while the least intervention was made on nonpharmacologic anticoagulant, elastic stockings. Table 2 of the 353 interventions made, adverse events $(32.86 \% 0$ was the highest followed by effectiveness of therapy $(18.70 \%)$, using laboratory indices of International Normalized Ratio (INR). The least determinant of intervention was drug-drug interaction of which $(2.27 \%)$ interventions were made. Figure 3 depicts the duration of admission in days. Here the shortest duration of admission was in most people (66.7\%) while the longest admission in a few patients (1.2\%).

Table 1: Anticoagulants on which intervention was made.

\begin{tabular}{|cccc|}
\hline SNO & $\begin{array}{c}\text { Types of } \\
\text { anticoagulation }\end{array}$ & $\begin{array}{c}\text { No of } \\
\text { interventions }\end{array}$ & Percentage (\%) \\
\hline 1 & Warfarin & 90 & 53.57 \\
2 & Enoxaparin & 59 & 35.11 \\
3 & Heparin & 5 & 2.9 \\
4 & Elastic stocking & 4 & 2.38 \\
5 & $\begin{array}{c}\text { Warfarin \& } \\
\text { Enoxaparin }\end{array}$ & 6 & 3.57 \\
Total & Warfarin \& Heparin & 10 & 5.95 \\
\hline
\end{tabular}

Table 2: Highlights the roles of a pharmacist.

\begin{tabular}{|cccc|}
\hline SNO & Determinants & $\begin{array}{c}\text { No of } \\
\text { interventions }\end{array}$ & Percentage (\%) \\
\hline 1 & $\begin{array}{c}\text { Effectiveness of } \\
\text { therapy (lab) } \\
\text { Drug-Drug } \\
\text { interaction }\end{array}$ & 66 & 18.7 \\
3 & Adverse events & 116 & 16.43 \\
4 & Dosage adjustments & 39 & 32.86 \\
5 & Kidney function & 6 & 11.05 \\
6 & Adherence to therapy & 60 & 16.7 \\
7 & Drug selection & 8 & 2.27 \\
8 & Total & 353 & 100 \\
\hline
\end{tabular}



Figure 1: Age range of patients admitted in ICU.

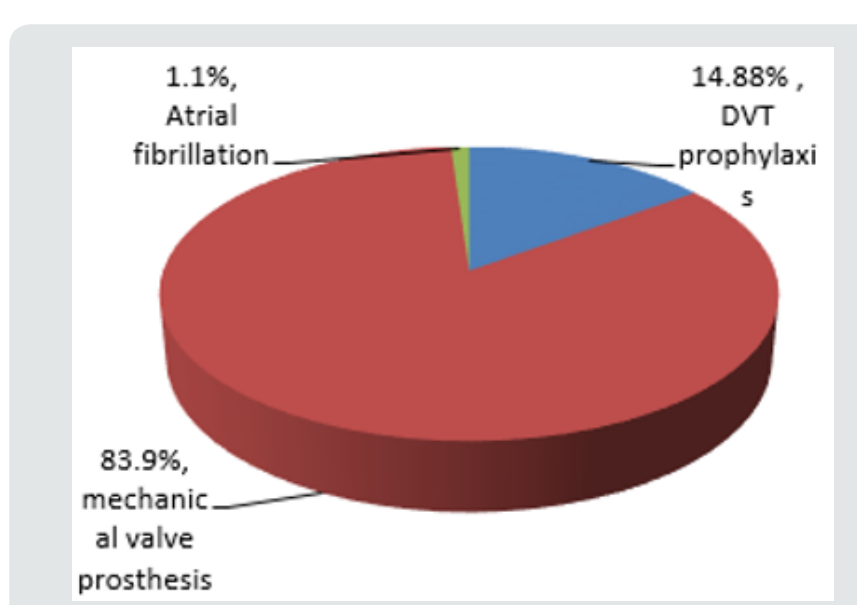

Figure 2: Indications for anticoagulation.

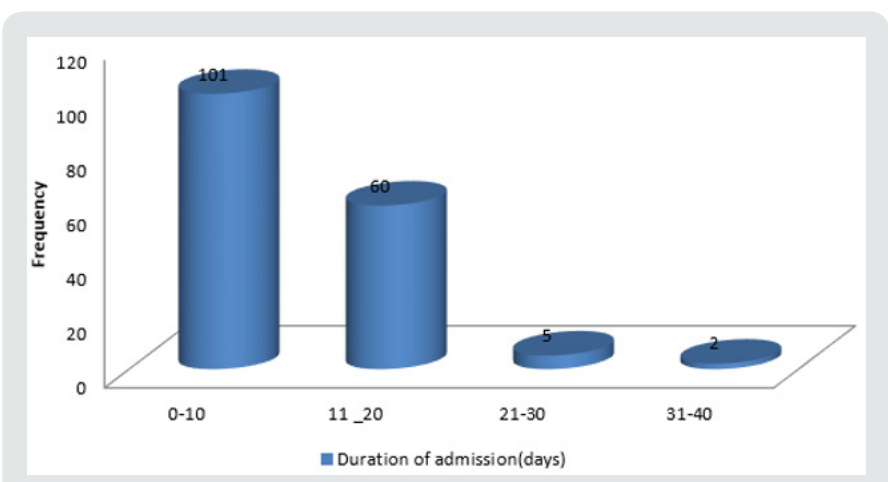

Figure 3: Duration of admission (days).

\section{Discussion}

Intensive care Unit (ICU) is a potential area for drug therapy problems (DTPs): patients treated are complex patients [19]. The use of thromboprophylaxis or prophylactic anticoagulant in critically ill patients is pertinent. ACCP recommends thromboprophylaxis for prevention of VTE in critical care patients (grade 1a - strong recommendation with high quality of evidence $[20,21]$. Omission of thromboprophylaxis within the first $24 \mathrm{hrs}$ of ICU admission without obvious reasons is associated with a higher risk of mortality [22]. Demographically, 168 patients admitted in ICU over 2 years (84-patients/year) had anticoagulation therapy. 
96 females and 72 males were involved with a ratio of $1.3: 1$. The age ranges were from 0-10 to 81-90 years with highest number that received anticoagulation being 31-40 years. The bulk of those with valve prostheses replacement came from this group. Duration of anticoagulation therapy paralleled relatively the duration of admission in ICU. In this study, the longest duration was 40 days while the least was 5 days. Overall, the average length of hospital stays amounted to 0.24 day/patient. Prospective evaluation of daily pharmacist-managed inpatient anticoagulation services was compared with a matched historical control group who received physician-managed anticoagulation in a 400-bed university teaching hospital [23]. *26A. Those who received pharmacistmanaged anticoagulation had significantly shorter hospital length of stay (6.8 days vs 9.5 days, P 5 .009), fewer supratherapeutic INR 3.5 (27\% vs $62 \%, \mathrm{P}, .001)$,Fewer readmission rates for bleeding or thrombosis within 1 to 3 months after discharge and no differences were noted between the groups INR ratio at discharge (2.6 vs 3.3, $\mathrm{P}$ 5.07 ) as well as number of bleeding complications (6 vs 1, P 5.11 ).

Pharmacists have been incorporated into ICU multiprofessional staff to improve the care provided to patients, particularly by monitoring the drugs administered and assessing their efficacy, thus contributing to improving patient safety [24]. The participation of clinical pharmacists in routine ICU care mainly includes active involvement in daily rounds, where they provide relevant information to the medical and nursing staff, analysis and monitoring of the efficacy of pharmacological treatments, implementation of medication reconciliation, and prevention, identification, and reporting of adverse reactions [25-27]. The actions performed by clinical pharmacists relative to the monitoring of pharmacological treatment are referred to as pharmacist interventions or recommendations (PhRs) [24,28]. Such professional interventions presuppose actions targeting pharmacological treatment to correct or prevent negative clinical outcomes derived from the use of medications. In the light of the above, the pharmacist role in the anticoagulant therapy for patients admitted in our institutional ICU was done. His/her actions in managing patients in anticoagulation in ICU in terms of monitoring of the pharmacological therapy of the anticoagulants are referred as interventions or recommendations. There have been several reports related to such interventions internationally in patients admitted in ICU [1,29]. Similar reports are rare in developing countries like Brazil including Nigeria. A retrospective chart review conducted at Baylor College in USA, in 2000 [30], showed four main variances related to warfarin therapy. They were inappropriate administration of warfarin loading dose, use of vitamin $\mathrm{K}$ when not recommended by American College of Chest Physicians guidelines, inconsistent overlapping of heparin with warfarin and inconsistent provision of patient education [30]. In this study, the determinants of the interventions ranged from effectiveness of therapy to drug selection, see Table 2. With this subgroup of effectiveness of therapy, international Normalized Ratio was used to monitor dosages which enabled the pharmacist to classify them as sub therapeutic, therapeutic and supratherapeutic.

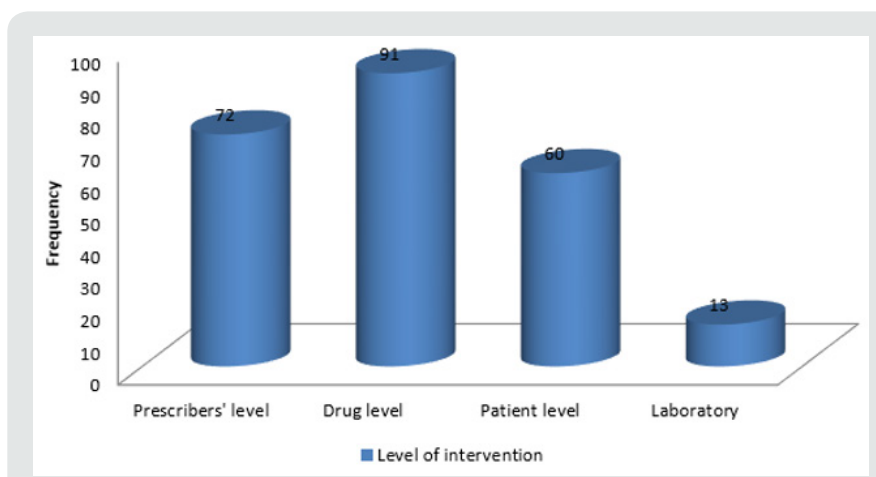

Figure 4: Level of intervention.



Figure 5: Acceptance of intervention.

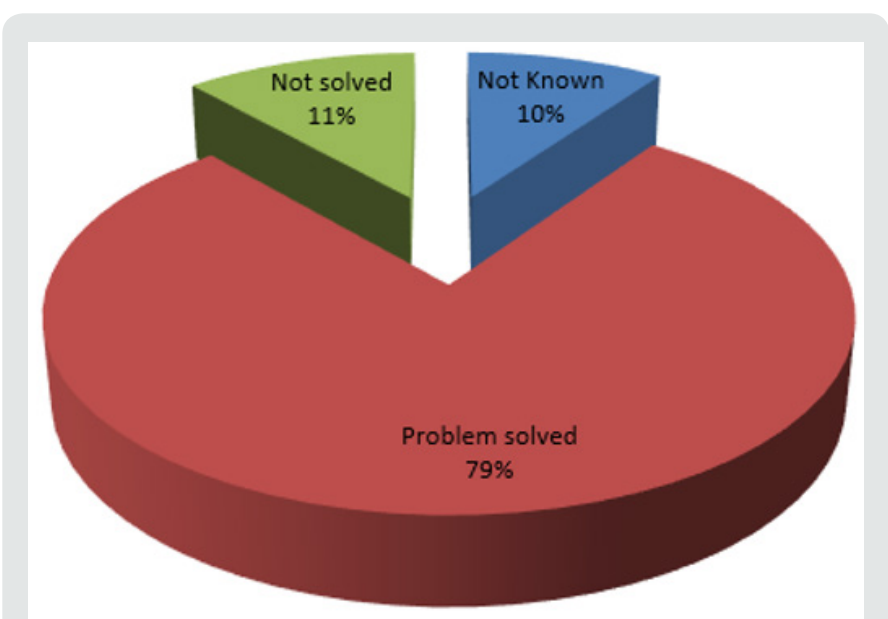

Figure 6: Outcome of intervention.

In this same scenario, drug-drug or drug-food interactions were monitored and classified as minor, significant or serious, see Table 3. When the effectiveness of therapy was supra or sub therapeutic, and when the DDI/DFIs were significant or serious, pharmacist intervened or made recommendations. The same scenario applied to adverse reactions, adherence to therapy as well as kidney function. These recommendations were made at multiple levels ranging from physicians/prescribers' level to laboratory level. In view of the fact this pharmacist's interventions or recommendations are novel in our institution and indeed in Nigeria, 
many interventions were accepted while some were rejected, see Figure 4. The outcome of such interventions is shown in Figures 5 \& 6 , indicating excellence when such recommendations are accepted. In a prospective, interventional study in a multidisciplinary ICU of a rural district hospital in Bathalapalli, Andhra Pradesh, India by Pichala et al. 72 patients were screened for DTPs, 947 drug doses prescribed, 148 prescriptions, 6.39 drugs per prescription and 13.15 drugs per patients of 243 DTPs identified, 1.67 DTPs present were prescriptions, drug interactions $78 \%$, inappropriate indication 7.4\%, ADRs and therapeutic duplications 2\%. $47 \%$ of the interventions were accepted. This was significantly lower when compared with our study. ICU SUCCESS DEDICATED PHARMACIST study done by Leape et al., concluded that: participation of the pharmacist on medical round can be a powerful means of reducing the risks of ADEs, in the ICU 99\% of pharmacist recommendations to medical staff were well accepted, an existing pharmacist intervention required no additional resources; instead it represented a different use of existing pharmacists' time.

Table 3: Determinants of pharmacist's roles.

\begin{tabular}{|c|c|c|c|c|c|}
\hline S NO & $\begin{array}{c}\text { Effectiveness of } \\
\text { therapy (laboratory) }\end{array}$ & $\begin{array}{l}\text { Drug-Drug } \\
\text { interaction }\end{array}$ & Adherence & Adverse events & Kidney function \\
\hline 1 & Therapeutic (28) & Minor (11) & Manifest problem (0) & Written education (30) & Serum creatinine (6) \\
\hline 2 & Subtherapeutic (32) & Significant (26) & $\begin{array}{c}\text { Potential problem } \\
\text { (116) }\end{array}$ & Verbal education (30) & \\
\hline 3 & Supratherapeutic (6) & Serious (21) & & Both (60) & \\
\hline
\end{tabular}

\section{Conclusion}

Anticoagulation treatment of patients in ICU requires precise dosing, a defined anticoagulant management programme, approved protocols, proper monitoring by clinical pharmacist (base line and follow-up INRs), education of patients and families about: dietary restrictions, monitoring follow up, drug interactions for outpatient and inpatient components. All these will result in improvement in the INR results, significant clinical outcomes and overall quality of care.

\section{Acknowledgement}

The authors wish to acknowledge the chairman NCTCE, Prof Basden Onwubere and the entire secretarial staff for their tremendous role in the production of this manuscript.

\section{References}

1. Prelaski CR, Lat I, Maclaren RM, Poston J (2013) Pharmacist contribution as members of the multidisciplinary ICU team. Chest 144(5): 1682-1695

2. Minet C, Lugos M, Savoye PY, Menez C, Ruckly S, et al. (2012) Pulmonary embolism in mechanically ventilated patients requiring computed tomography: prevalence, risk factors and outcome. Critical Care Med 40(12): 320-328

3. Geerts WH, Beegqust D, Pineo GF, Heit JA, Samama CM, et al. (2008) Prevention of Venous thromboembolism. American College of Chest Physician -Evidence based Clinical Practice Guidelines ( $8^{\text {th }}$ edn). Chest 135: 3815-4535.

4. Shorr AF, Williams MD (2009) Venous Thromboembolism in critically ill patients. Observations from a randomized trial in sepsis. Thromb Haemost 101(1): 139-144.

5. Cook D, Attia J, Weaver B, McDonald JE, Crowther M (2000) Venous thromboembolic disease: an observational study in medical-surgical intensive care unit patients. J Critical Care 15(4): 127-132.

6. Timsit JF, Farkas JC, Bayer JM, Martin JB, Misset B, et al. (1981) Central Venous Catheter related thrombosis in intensive care patients: incidence, risk factorsand relationship with catheter related sepsis. Chest 114(1): 207-213.

7. Chartre J, Cormid J, Bourchama A, Vian F, Benacenat R, et al. (1982) Thrombosis as a complication of pulmonary artery catheterization via the Internal Jugular Vein: Prospective evaluation by phlebography. New Eng J Med 306(5): 278-281.

8. Joynt GM, Kav J, Gomersall CD, Leung VY, Liu ER (2000) Deep Venous thrombosis caused by femoral veins catheter in critically ill adult patients. Chest 117(1): 178-183.

9. Trottier SJ, Veremakis C, O Brien J, Auer AI (1995) Femoral Deep Vein Thrombosis associated with cental venous catheterization: results from a prospective randomized trial. Crit Care Med 23(1): 52-59.

10. Read II, Luna M, Khalil SA, Costentin JW, Ram C, et al. (1994) The relationship between the thrombotic and infectious complications of central venous catheter. JAMA 271: 104-106.

11. Dorffler Melly J, de Jonge E, Pont AC, Meljeis J, Vroom MD, et al. (2002) Bioavailability of subcutaneous Lower Molecular Heparin in patients on vasopressors. Lancet 359(9309): 849-850.

12. Selby R, Geerts W (2009) Prevention of Venous Thromboembolism: consensus, controversies and challenges. Hematology program 2009: 286-292.

13. Kulik A, Ruben FD, Well PS, Kearon C, Mesoma TG, et al. (2006) Early postoperative anticoagulation after mechanical valve replacement: a systematic review. Ann thorac Surg 81(2): 770-781.

14. John S, Ravikumar E, John CN, Bashi VV (2000) 25-year experience with combined mitral and aortic valve replacement for rheumatic heart disease. Ann Thorac Surg 69(4): 1167-1172.

15. Saxena R, Lewis S, Berge E, Sanderasck PA, Kondstaal PJ (2001) Risk of early death and recurrent stroke and effect of heparin in 3169 patients with acute ischemic stroke and arial fibrillation in the international stroke trial. Stroke 32(10): 2333-2337.

16. Lin HJ, Wolf PA, Kelly Hayes M, Bieser AS, Kase CS, et al. (1996) Stroke severity in atrial fibrillation: the Framingham study. Stroke 27(10): 1760-1764.

17. Investigation Atrial fibrillation (1994) Risk factors for stroke and efficacy of antithrombotic therapy in Atrial fibrillation. Analysis of pooled data from five randomized trials. Arch Intern Med 154: 1449-1457.

18. Tunic PA, Nayar AC, Goodkin GM, Mirchandani S, Francescone S, et al. (2002) Effect of treatment on the incidence of stroke and other emboli in 519 patients with severe thoracic aortic plaque. Am J Cardiol 90(12): 1320-1305.

19. Geerts WH, Bergqvist D, Pineo GF, Heit JA, Samama CM, et al. (2008) Prevention of venous thromboembolism: American College of Chest Physicians Evidence-Based Clinical Practice Guidelines ( $8^{\text {th }}$ edn $)$. Chest 133: 381S-453S. 
20. Kearon C, Akl EA, Comerota AJ, Prandoni P, Bounameaux H, et al. (2012) Antithrombotic therapy for VTE disease: Antithrombotic Therapy and Prevention of Thrombosis, ( $9^{\text {th }}$ edn): American College of Chest Physicians Evidence-Based Clinical Practice Guidelines. Chest 141: e419S-94S.

21. Ho KM, Chavan S, Pilcher D (2011) Omission of early thromboprophylaxis and mortality in critically ill patients: a multicenter registry study. Chest 140(6): 1436-1446.

22. Claus BO, Robays H, Decruyenaere J, Annemans L (2014) Expected net benefit of clinical pharmacy in intensive care medicine: a randomized interventional comparative trial with matched before-and-after groups. J Eval Clin Pract 20(6): 1172-1179.

23. Chisholm Burns MA, Kim Lee J, Spivey CA, Slack M, Herrier RN, et al. (2010) US pharmacists' effect as team members on patient care: systematic review and meta-analyses. Med Care 48(10): 923-933.

24. Klopotowska JE, Kuiper R, van Kan HJ, de Pont AC, Dijkgraaf MG, et al. (2010) On-ward participation of a hospital pharmacist in a Dutch intensive care unit reduces prescribing errors and related patient harm: an intervention study. Crit Care 14(5): R174.
25. Penm J, Chaar B, Rose G, Moles R (2015) Pharmacists' influences on prescribing: validating a clinical pharmacy services survey in the Western Pacific Region. Res Social Adm Pharm 11(1): 63-73.

26. Lee AJ, Boro MS, Knapp KK, Meier JL, Korman NE (2002) Clinical and economic outcomes of pharmacist recommendations in a Veterans Affairs medical center. Am J Health Syst Pharm 59(21): 2070-2077.

27. Urtaine I, Bandere D, Ansberge J, Stokmane A (2012) Role of Clinical Pharmacist in Anticoagulation Management. Medianos teorija Praktika 18: 189-192.

28. Damaseki EL, Baird RW (2005) Development and implementation of a Pharmacist managed inpatient warfarin protocol. Bayl Univ Med Cent 18(4): 397-400.

29. Pitchale, Kuma BM, Seeba Z (2013) An Interventional study on Intensive Care Unit drug therapy assessment in rural district hospital in India. Journal of Basic and Clinical pharmacy 4(3): 64-67.

30. Leap L, Cullen D, Clapp M (1993) Pharmacist Participation on Physician rounds and adverse drug events in the intensive care unit. JAMA 282(3): 267-270.
(C) This work is licensed under Creative

To Submit Your Article Click Here: Submit Article

DOI: $10.32474 /$ ACR.2019.02.000126

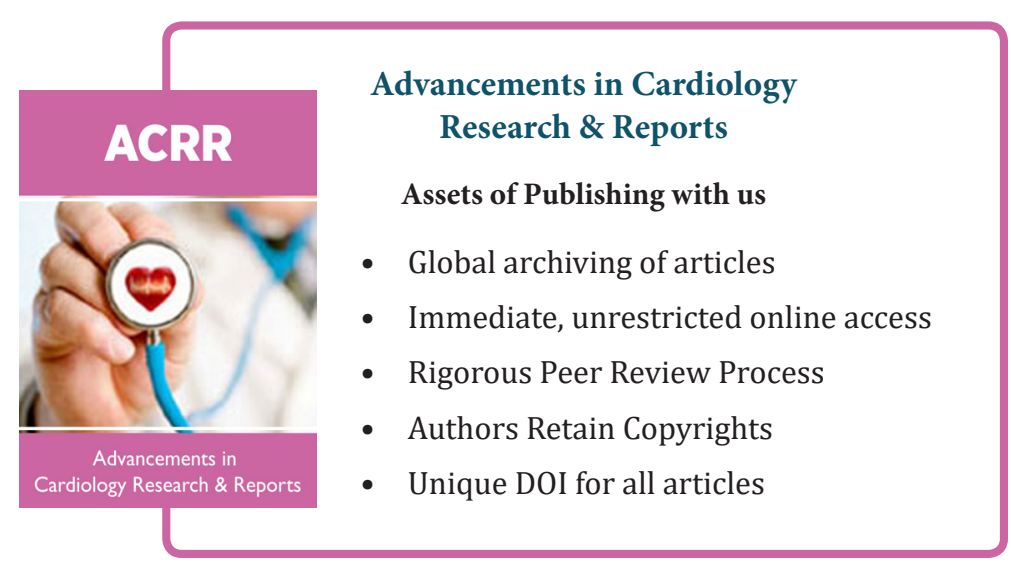

